\title{
Inhibition of angiogenesis by the poly(ADP-ribose) polymerase inhibitor PJ-34
}

\author{
ANASTASIA PYRIOCHOU ${ }^{1}$, GABOR OLAH $^{2}$, EDWIN A. DEITCH $^{2}$, \\ CSABA SZABÓ $^{2}$ and ANDREAS PAPAPETROPOULOS ${ }^{1}$ \\ ${ }^{1}$ Laboratory of Molecular Pharmacology, Department of Pharmacy, University of Patras, Patras, Greece; \\ ${ }^{2}$ Department of Surgery, University of Medicine and Dentistry of New Jersey, NJ, USA
}

Received January 10, 2008; Accepted February 20, 2008

\begin{abstract}
Angiogenesis-related treatments have a broad spectrum of potential applications ranging from cancer to macular degeneration, to wound healing. Thus, the identification of pharmacological agents that modulate new blood vessel formation has attracted much attention. In the present study, we investigated the effects of the poly(ADP-ribose) polymerase (PARP) inhibitor PJ-34 [N-(6-Oxo-5,6-dihydro-phenanthridin-2-yl)-N,N-dimethylacetamide] on angiogenesis. Treatment of chicken chorioallantoic membranes (CAM) with PJ-34 reduced vascular length in these tissues; paradoxically, lower doses of PJ-34 (0.03 or $\left.0.3 \mathrm{nmol} / \mathrm{cm}^{2}\right)$ were more effective in inhibiting neovascularisation than higher doses ( 3 or $30 \mathrm{nmol} / \mathrm{cm}^{2}$ ). In vitro, incubation of endothelial cells (EC) with PJ-34 (300 nM to $10 \mu \mathrm{M})$ inhibited their proliferation in a concentration-dependent manner with maximal inhibition of $22.3 \%$ being observed at $10 \mu \mathrm{M}$. Capillary morphogenesis of EC grown on Matrigel was also negatively affected by PJ-34. In addition, PJ-34 abolished the migratory response to the prototype angiogenic factor vascular endothelial growth factor (VEGF) and reduced VEGFstimulated activation of members of the mitogen activated protein kinase family (ERK1/2, p38), as well as Akt. PJ-34 also inhibited VEGF-induced NO release and cGMP
\end{abstract}

Correspondence to: Dr Andreas Papapetropoulos, Laboratory of Molecular Pharmacology, Department of Pharmacy, University of Patras, 26504 Patras, Greece

E-mail: apapapet@upatras.gr

Abbreviations: CAM, chorioallantoic membrane; EC, endothelial cells; ECGS, endothelial cell growth supplement; ERK1/2, extracellular signal regulated kinase; HUVEC, human umbilical vein endothelial cells; IBMX, isobutylmethylxanthine; MAPK, mitogen activated protein kinase; NO, nitric oxide; PI3-K, phosphoinositide 3 kinase; sGC, soluble guanylyl cyclase; PARP, poly(ADP-ribose) polymerase; PJ-34, [N-(6-Oxo-5,6-dihydrophenanthridin-2-yl)-N,N-dimethylacetamide]; VEGF, vascular endothelial growth factor

Key words: angiogenesis, poly(ADP-ribose), polymerase inhibitor accumulation. In conclusion, we provide evidence that PARP inhibition blocks angiogenesis-related EC properties by interfering with multiple signalling pathways leading to the inhibition of new blood vessel formation.

\section{Introduction}

In adult organisms the formation of new blood vessels from pre-existing structures, termed angiogenesis, is a tightly regulated process $(1,2)$. Physiological processes and functions, such as wound healing, endometrial hyperplasia during the menstrual cycle and increase in skeletal muscle mass during exercise training all require an active angiogenic response (3). On the other hand, uncontrolled angiogenesis has been implicated in pathological phenomena; these include tumor growth, psoriasis, arthritis and diabetic retinopathy $(3,4)$. For new blood vessels to form, endothelial cells (EC) need to degrade their extracellular matrix, proliferate, migrate and differentiate into patent structures; mural cells are then recruited to structurally support the new structures formed (1).

PARP-1, the most abundant member of the poly(ADPribose) polymerase (PARP) family, catalyzes the post-translational modification of proteins using $\mathrm{NAD}^{+}$as a substrate. One of the main stimuli for PARP-1 activation is the presence of breaks in the DNA strands (5). The activation of PARP-1 is crucial for DNA repair; however, when massive DNA damage has occurred, PARP-1 induces cell death rather than survival due to the depletion of cellular $\mathrm{NAD}^{+}(6)$. Alternative pathways, unrelated to DNA damage for PARP-1 activation, have been described and include direct interaction with phosphorylated ERK2 (7) and increased intracellular calcium levels (8). Apart from DNA repair and cell death, PARP-1 has been shown to affect cell cycle progression and to contribute to the regulation of gene expression $(5,9)$. Poly(ADP-ribosyl)ation of histones induces chromatin relaxation, allowing transcription factors to access DNA; PARP also participates in promoter/enhancer binding complexes $(5,9)$. On the other hand, poly(ADP-ribosyl)ation of a number of transcription factors prevents their binding to DNA and decreases their transcriptional activity (10).

Recently, it was shown that VEGF protein synthesis is increased in EC by inhibiting poly(ADP-ribosyl)ation, suggesting that PARP inhibitors might enhance angiogenesis (11). In contrast, the pharmacological inhibition of PARP 
was shown to inhibit angiogenic sprout formation and the growth factor-stimulated migration and proliferation of EC $(12,13)$. To determine whether inhibition of PARP modifies angiogenesis in vivo, we used a well-established angiogenesis assay, the chicken chorioallantoic membrane assay. Furthermore, we evaluated the contribution of PARP activation to the angiogenic actions of VEGF and studied the ability of PJ-34 to inhibit VEGF-stimulated signalling and the VEGFtriggered angiogenesis-related properties of EC.

\section{Materials and methods}

Materials. Fertilized white leghorn chicken eggs were obtained from Pindos (Iperos, Greece). Cell culture media and serum were obtained from Life Technologies Gibco-BRL (Paisley, UK). All cell culture plastic ware was purchased from Corning-Costar Inc. (Corning, NY); SuperSignal West Pico chemiluminescent substrate from Pierce Biotechnology (Rockford, Illinois); DC Protein Assay kit, Tween-20 and other immunoblotting reagents were obtained from Bio-Rad Laboratories (Hercules, CA); penicillin and streptomycin from Applichem (Darmstadt, Germany); amphotericin, gentamycin and heparin were purchased from Biochrom AG (Berlin, Germany). Reduced growth factor Matrigel was obtained from BD Biosciences (Bedford, MA). VEGF was purchased from Peprotech (London, UK). The cGMP low $\mathrm{pH}$ kit was obtained from Assay Design (Ann Arbor, MI). The PARP inhibitor was a gift from Inotek Corporation (Beverly, MA). ERK1/2, p38 and Akt phospho-specific and total antibodies along with the secondary antibodies were obtained from Cell Signaling Technology (Beverly, MA). All other reagents including bovine serum albumin, DETA-NO, BAY 41-2272 and protease inhibitors were purchased from SigmaAldrich (St. Louis, MO).

In vivo CAM angiogenesis assay. Fertilised white leghorn chicken eggs were placed in an incubator and kept under constant humidity at $37^{\circ} \mathrm{C}$. On day 4 , a square window was opened in the shell and then sealed with adhesive tape. On day 9 , an $\mathrm{O}$ ring $\left(1 \mathrm{~cm}^{2}\right)$ was placed on the surface of the CAM and PJ-34 at 0.03 to $3 \mathrm{nmol} / \mathrm{cm}^{2}$ or vehicle were added inside this restricted area. After $48 \mathrm{~h}, \mathrm{CAMs}$ were fixed in Carson's solution and angiogenesis was evaluated using the NIH image analysis software.

Cell culture and proliferation. Human umbilical vein endothelial cells (HUVEC) were isolated from 2-4 fresh umbilical cords and grown on 100-mm dishes in M199 supplemented with $15 \%$ fetal calf serum, $50 \mathrm{U} / \mathrm{mL}$ penicillin and $50 \mu \mathrm{g} / \mathrm{mL}$ streptomycin, $50 \mu \mathrm{g} / \mathrm{ml}$ gentamycin, $2.5 \mu \mathrm{g} / \mathrm{ml}$ amphotericin B, $5 \mathrm{U} / \mathrm{ml}$ sodium heparin and 150-200 $\mu \mathrm{g} / \mathrm{ml}$ endothelial cell growth supplement (ECGS). HUVEC between passages 2 and 3 were used for all experiments. The starvation medium lacked serum and ECGS as opposed to the complete medium. For the proliferation assays, endothelial cells (EC) were seeded in 24-well plates at 6,000 cells $/ \mathrm{cm}^{2}$, in complete growth medium, and allowed to adhere overnight. They were then exposed to PJ-34 $(0.3-10 \mu \mathrm{M})$ or vehicle (DMSO) for $48 \mathrm{~h}$. After this time, the cells were trypsinized and the cell number was determined using a hemocytometer.
Matrigel morphogenetic assay. HUVEC were plated at 15,000 cells/well in 96-well plates, pre-coated with $50 \mu \mathrm{L}$ of growth factor-reduced Matrigel matrix in the presence or absence of PJ-34 $(0.3 \mu \mathrm{M})$. After $24 \mathrm{~h}$ of incubation, tubelike structure formation was quantified using image analysis software.

Cell migration. HUVEC were serum starved overnight. To inhibit PARP, the cells were pre-treated with PJ-34 $(0.3 \mu \mathrm{M})$ for 15 min prior to trypsinization. After trypsinization, $1 \times 10^{5}$ cells were added to transwells $(8-\mu \mathrm{M}$ pore size) in $100 \mu 1$ of starvation medium. The test articles including PJ-34 $(0.3 \mu \mathrm{M})$, VEGF $(50 \mathrm{ng} / \mathrm{ml})$, the NO-independent activator of soluble guanylyl cyclase (sGC) BAY 41-2272 $(0.1 \mu \mathrm{M})$, the NO donor DETA-NO $(100 \mu \mathrm{M})$ or vehicle (DMSO) were added to the well containing the transwell inserts at $600 \mu \mathrm{L}$ volume. PJ-34 was added in both the upper and lower compartments of the transwell setup. EC were allowed to migrate for $4 \mathrm{~h}$ at $37^{\circ} \mathrm{C}$. Non-migrated cells at the top of the transwell filter were removed with a cotton swab. The migrated cells were fixed in Carson's solution (30 min at room temperature) and then stained in toluidine blue (20 $\mathrm{min}$ at room temperature). Migrated cells were scored in 8 random fields and the foldchange was determined compared to the number of migrated cells in control wells.

Cell lysate preparation and Western blotting. After the treatments, proteins from HUVEC were extracted after homogenization in a lysis buffer containing $1 \%$ Triton-X, $1 \%$ SDS, $150 \mathrm{mM} \mathrm{NaCl}, 50 \mathrm{mM} \mathrm{NaF}, 1 \mathrm{mM} \mathrm{Na} \mathrm{VO}_{4}, 0.5 \%$ sodium deoxycholate, $1 \mathrm{mM}$ EDTA, $0.1 \mathrm{mM}$ EGTA and protease inhibitors $(10 \mu \mathrm{g} / \mathrm{ml}$ aprotinin, $10 \mu \mathrm{g} / \mathrm{ml}$ pepstatin and $20 \mathrm{mM}$ PMSF). Samples were subjected to SDS-PAGE, transferred to an activated PVDF membrane, and incubated with the indicated primary and appropriate secondary antibodies. Immunoreactive proteins were detected using a chemiluminescent substrate.

cGMP enzyme immunoassay. Confluent monolayers of HUVEC were washed twice with Hanks' balanced salt solution and pre-treated with vehicle or PJ-34 (300 nM) for $60 \mathrm{~min}$. They were then stimulated for $15 \mathrm{~min}$ with VEGF $(50 \mathrm{ng} / \mathrm{ml})$ in Hanks' containing the phosphodiesterase inhibitor isobutylmethylxanthine $(1 \mathrm{mM})$. Cells were then lyzed in $0.1 \mathrm{~N} \mathrm{HCl}$ and cGMP content was measured in the supernatants using a commercially available enzyme immunoassay kit following the manufacturer's instructions.

Data analysis. Data are expressed as means \pm SEM of the indicated number of observations. Statistical comparisons between groups were performed using ANOVA or Student's t-test, as appropriate. Differences were considered significant when $\mathrm{p}<0.05$.

\section{Results}

PARP promotes capillary morphogenesis in vitro and neovascularisation in vivo. In order to test whether PARP contributes to the formation of new blood vessels in vivo, we used the pharmacological inhibitor PJ-34 (14) in the chicken 


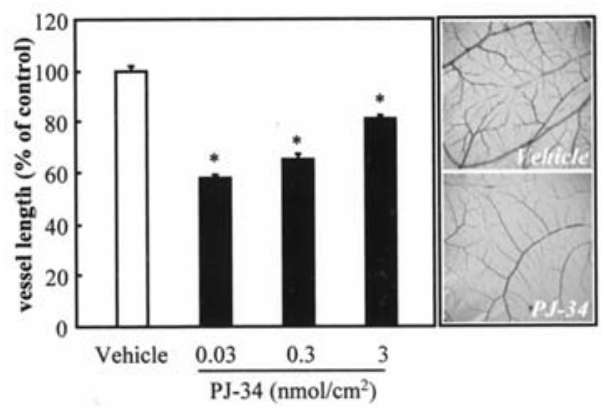

Figure 1. Inhibition of PARP attenuates angiogenesis in vivo. CAMs were treated with the indicated dose of PJ-34 for $48 \mathrm{~h}$; the total length of vessel network was determined using image analysis software. A representative photomicrograph (lower right) shows reduction in angiogenesis following PJ-34 treatment. Data are expressed as means \pm SEM; $n=22-32 ;{ }^{*} \mathrm{p}<0.05$ vs. vehicle.

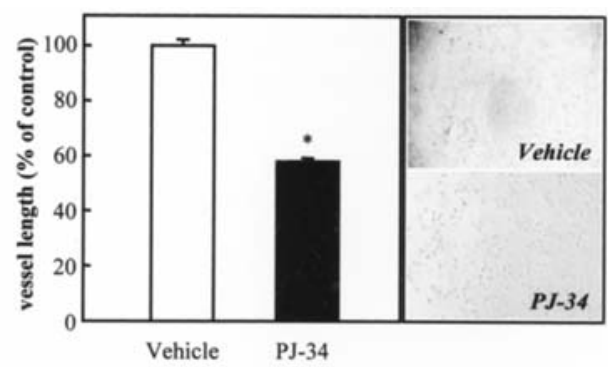

Figure 2. PJ-34 blocks tube-like network formation of endothelial cells. HUVEC were cultured on growth factor-reduced Matrigel in the presence of vehicle (DMSO) or PJ-34 (300 nM) for $24 \mathrm{~h}$. Network length was then determined using image analysis software. Representative photomicrographs (right) showing reduction of network formation. Data are expressed as means \pm SEM; $n=6 ;{ }^{*} \mathrm{p}<0.05$ vs. vehicle.

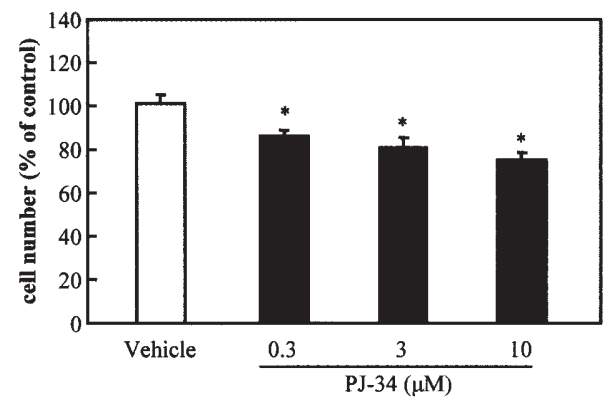

Figure 3. PARP inhibition decreases endothelial cell growth. HUVEC were plated, allowed to adhere and incubated with vehicle or PJ-34 $(0.3,3$ or $10 \mu \mathrm{M})$ for $48 \mathrm{~h}$ in growth medium. Cell number was then determined by a hemocytometer. Data are expressed as means \pm SEM; $n=4 ;{ }^{*} p<0.05$ vs. vehicle.

chorioallantoic membrane (CAM) model of angiogenesis. The length of the vascular network in the CAM decreased in response to PJ-34 treatment; however, PJ-34 exhibited an inverse dose-response with the lower dose of PJ-34 being more effective in inhibiting angiogenesis than the higher ones (Fig. 1). When doses $>3 \mathrm{nmol}$ were used, the results obtained were similar to those observed at $3 \mathrm{nmol}$ (data not
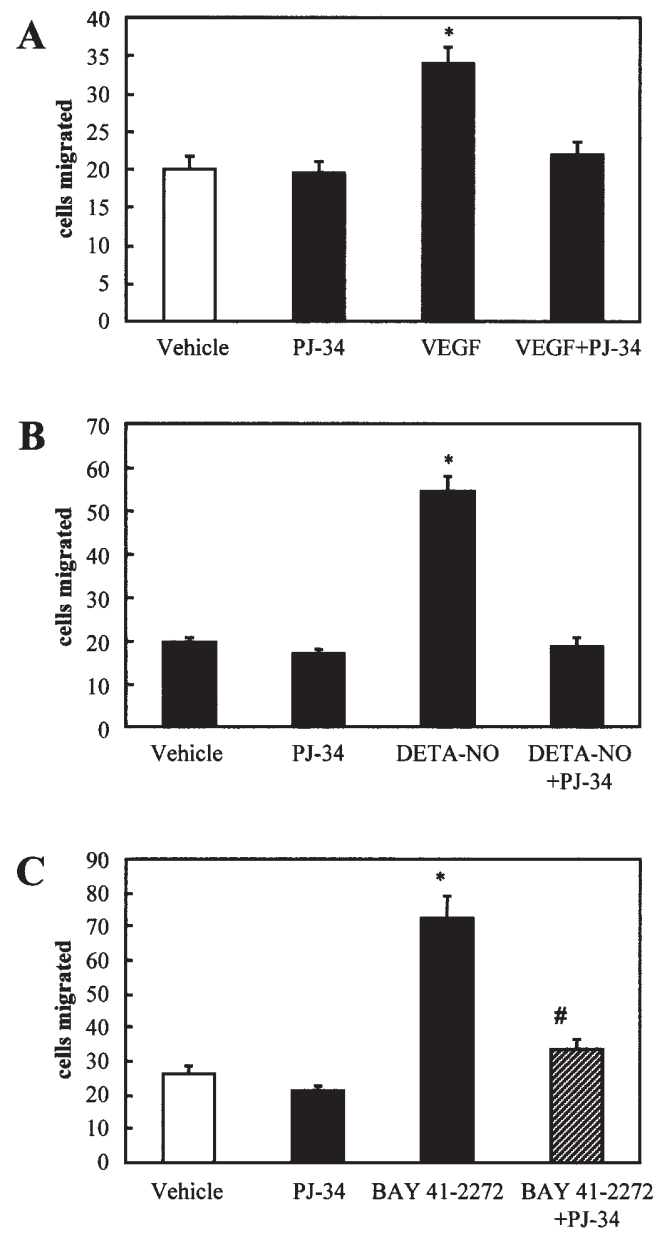

Figure 4. PARP inhibition abrogates VEGF- and sGC-stimulated EC migration. HUVEC were serum starved overnight and then treated with PJ-34 ( $300 \mathrm{nM}$ ) for $30 \mathrm{~min}$. Cells were then trypsinized, placed in transwells in the absence or presence of PJ-34 (300 nM) and allowed to migrate for $4 \mathrm{~h}$ in response to (A) VEGF $(50 \mathrm{ng} / \mathrm{ml})$, (B) DETA-NO $(10 \mu \mathrm{M})$ or (C) BAY 41$2272(0.1 \mu \mathrm{M})$. Data are expressed as means $\pm \mathrm{SEM} ; \mathrm{n}=5 ;{ }^{*} \mathrm{p}<0.05$ vs. vehicle; ${ }^{\#} \mathrm{p}<0.05$ vs. PJ-34.

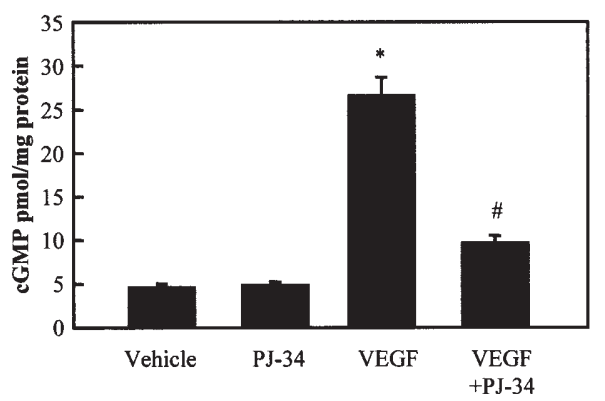

Figure 5. PARP inhibition blocks cGMP formation. HUVEC were pretreated with PJ-34 (300 nM) for $30 \mathrm{~min}$. Cells were then exposed for $15 \mathrm{~min}$ to VEGF $(50 \mathrm{ng} / \mathrm{ml})$ in the presence of the phosphodiesterase inhibitor IBMX (1 mM); cGMP was extracted using $0.1 \mathrm{~N} \mathrm{HCl}$ and measured by enzyme immunoassay. Data are expressed as means \pm SEM; $n=4 ;{ }^{*} p<0.05$ vs. vehicle " $\mathrm{p}<0.05$ vs VEGF.

shown). To study the ability of PJ-34 to interfere with tubelike structure formation in vitro, EC were cultured on Matrigel and network length formation was determined. Results from these experiments showed that the inhibition of PARP blocks 

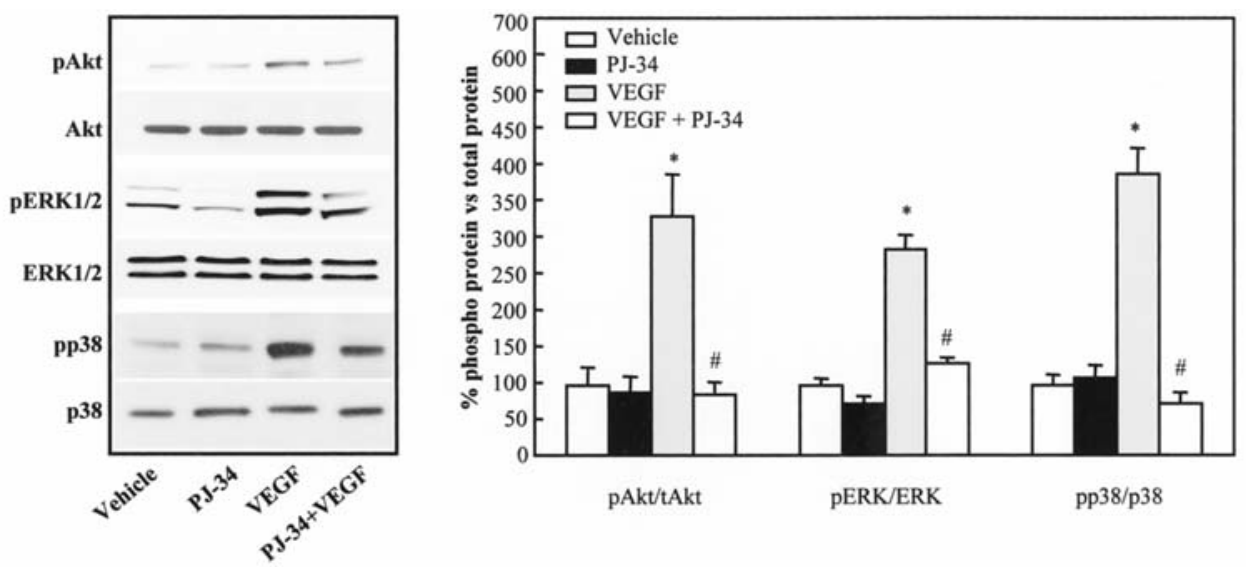

Figure 6. PJ-34 inhibits VEGF-triggered signalling. HUVEC were serum starved for $4 \mathrm{~h}$ and pre-treated with PJ-34 (300 $\mathrm{nM}$ ) for $30 \mathrm{~min}$. They were then stimulated with VEGF (50 ng/ml) for $15 \mathrm{~min}$. After the incubation, lysates were prepared, samples subjected to SDS-PAGE and membranes were incubated with the indicated primary antibodies. Following incubation with the secondary antibody, immunoreactive bands were visualized using a chemiluminescent substrate. Blots shown are representative of experiments performed three times.

the characteristic changes in morphology of EC induced by Matrigel (Fig. 2).

PARP inhibition attenuates endothelial proliferation and migration. To evaluate the effect of PARP on cell growth, HUVEC were allowed to proliferate in complete medium and their number determined in the presence or absence of PJ-34 after 48 h. PJ-34 reduced EC number in a concentrationdependent manner (Fig. 3). It should be noted that PJ-34 does not reduce cell viability in HUVEC at a concentration up to $6 \mu \mathrm{M}$ (12). We next examined the effect of PJ-34 on EC migration. For these studies, the prototype angiogenic factor VEGF, was used to drive the migratory response. While stimulation of HUVEC with VEGF increased their migratory rate by approximately 2-fold, blockade of PARP inhibited the VEGF-stimulated migration (Fig. 4A). As activation of nitric oxide and soluble guanylyl cyclase activation are known to mediate the effects of VEGF with respect to migration (15), we treated EC with a $\mathrm{NO}$ donor or a NO-independent activator of sGC (BAY 41-2272) and studied the effect of PJ-34 on EC motility. In line with previous observations, both agents increased HUVEC migration (Fig. 4B and C). Similarly to what was observed with VEGF, PARP inhibition abolished the DETA-NO- and BAY 4122-72-induced responses. To determine the ability of PARP to modulate VEGF-induced activation of the NO/cGMP pathway, we tested the effect of PJ-34 on cGMP accumulation. Stimulation of HUVEC with VEGF enhanced the intracellular levels of cGMP by more than 5-fold (Fig. 5). Pre-treatment with PJ-34 blocked the VEGF-induced cGMP production, suggesting that PARP activity is essential for $\mathrm{NO}$ production and/or sGC activation.

Role of PARP in VEGF-stimulated kinase pathways. To examine the molecular mechanisms through which PJ-34 inhibits the angiogenesis-related properties of EC we focused on the activation of kinases known to be important in VEGFstimulated angiogenesis, namely Akt, ERK1/2 and p38 MAPK. While exposure of cells to VEGF enhanced the phosphorylation of all three kinases tested, pre-treatment with PJ-34 reduced the VEGF responses, suggesting that PARP is required for VEGF signalling (Fig. 6).

\section{Discussion}

The main findings of the present study are that, i) inhibition of PARP reduces neovascularization in vivo, ii) inhibition of PARP attenuates angiogenesis-related properties of EC in vitro, iii) pre-treatment of cells with PJ-34 abolishes the VEGF-induced activation of MAPK and Akt, and iv) PJ-34 reduces VEGF-stimulated NO production and cGMP accumulation.

PARP-1 has been shown to play an important role in the repair of DNA strand breaks caused by ionizing radiation and alkylating agents $(16,17)$. Thus, the ability of PARP-1 inhibitors to enhance the toxicity of radiotherapy or chemotherapy on tumors, is currently being evaluated in phase I/II clinical trials (18). As most tumors do not grow beyond a few millimeters without an increase in their vascularity (2), we evaluated whether PARP inhibitors affect endothelial, as well as cancer, cells. To determine the effects of PARP inhibition with respect to angiogenesis we evaluated the ability of PJ-34 to block new blood vessel formation. Treatment of CAM with PJ-34 led to reduced vascular network length. However, PJ-34 exerted an inverse dose-dependent effect, with the lowest dose used being the most effective in inhibiting angiogenesis. The inverse dose-response exhibited by PJ-34 might be explained by the fact that PARP regulates the activity of multiple signalling pathways and transcription factors that are likely to exert opposing effects on blood vessel formation. Lower PJ-34 might preferentially inhibit pathways leading to the expression of angiogenic factors or induce the production of endogenous angiogenesis inhibitors, while as PJ-34 concentrations increase, inhibitory pathways are switched off. Our findings that PARP inhibition reduces neovascularization in the CAM are in line with the observation that PARP-1 deletion or administration of a PARP inhibitor (GPI15427) attenuates VEGF-driven angiogenesis in the Matrigel plug assay in mice (19).

We next studied the impact of PARP inhibition on angiogenesis-related properties of EC and determined the effects of PJ-34 on growth, migration and tube-like network formation. Incubation of cells with PJ-34 led to reduced proliferation. Our findings are in line with those of Rajesh et al demonstra- 
ting that PARP inhibitors block VEGF- and fibroblast growth factor-induced BrdU incorporation in EC $(12,13)$. It should be noted that PJ-34 was found to exert its anti-proliferative effect at lower concentrations than those already reported. In addition to its effects on growth, PJ-34 blocked matrix-driven tube-like structure formation. Our findings confirm and extend recent observations that PARP inhibition reduces growth factor-stimulated sprouting from aortic explants and network formation on Matrigel $(12,13)$. Moreover, to determine the effect of PJ-34 on cell motility, we pre-treated cells with the inhibitor and monitored their migration in response to VEGF. Under the conditions used, PARP inhibition abolished VEGF-driven migration. As earlier studies have indicated that PARP inhibition attenuates VEGF- and fibroblast growth factor-, but not epidermal growth factorinduced migration $(13,19)$, PARP inhibition might selectively affect certain angiogenic pathways.

Although the ability of PARP inhibitors to interfere with angiogenesis-related EC properties was previously suggested, no information concerning their mechanism of action is available. So far, only negative data is available showing that the PARP inhibitor GPI 15427, does not block cobalt-induced hypoxia inducible factor-1 activation (19). In our study we focused on MAPK kinase and PI3-K/Akt pathways that are known to regulate most aspects of the angiogenic response (proliferation, migration and differentiation) and have been shown to mediate the angiogenic properties of many growth factors. Our data showed that PJ-34 abolished VEGF-triggered phosphorylation of ERK1/2, p38 and Akt, providing a molecular basis for its anti-angiogenic effects. Given the critical role of p38 in VEGF-stimulated migration (20-22), it is tempting to speculate that PJ-34 exerted an inhibitory effect on VEGF-stimulated migration by blocking VEGF-triggered p38 activation. In addition, the ability of PJ-34 to block ERK $1 / 2$ is in agreement with the anti-proliferative actions exerted by this inhibitor. In line with our results, it has been shown that PJ-34 inhibits LPS-induced MAPK activation; however, in this setting PARP inhibition activated PI3-K/Akt $(23,24)$.

It is well-accepted that $\mathrm{NO}$ contributes to the angiogenic actions of VEGF $(25,26)$. We have shown that VEGFstimulated migration in HUVEC depends on the activation of the NO/cGMP pathway that in turn activates p38, leading to increased motility $(15,27)$. To further characterize the level at which PARP exerts its inhibitory effect on VEGF signalling, we determined the ability of PJ-34 to reduce NO-donor and cGMP-mediated migration and VEGF-triggered cGMP synthesis. Fulton et al previously showed that the exposure of EC to VEGF stimulates endothelial NO synthase phosphorylation on ser-1177 and activation that is mediated by PI3$\mathrm{K} / \mathrm{Akt}$ (28). Increased NO production then leads to sGC stimulation and increased cGMP synthesis (29). In concert with the ability of PJ-34 to block Akt activation, the pretreatment of cells with this PARP inhibitor attenuated VEGFstimulated cGMP formation. It should be noted that PARP inhibitors have been reported to block the expression of the inducible isoform of NO synthase (30). As VEGF-stimulated angiogenesis depends on both iNOS and eNOS activation (31), the anti-angiogenic effect of PJ-34 in vivo might be related to generalized suppression of NO production/cGMP accumulation. Interestingly, PJ-34 also inhibited BAY 412272-induced migration, indicating that PARP is required not only for cGMP accumulation, but also participates in downstream events leading to the activation of MAPK effectors. A direct interaction of ERK2 with PARP-1 that dramatically amplifies the downstream actions of ERK has already been shown to occur (7). Our data taken together suggest that PARP interferes with VEGF signalling at multiple levels.

In summary, we have shown that PJ-34 inhibits angiogenesis and blocks VEGF signalling. Given the fact that neutralizing the action of VEGF has proven to be successful in combating cancer in patients, the identification of novel inhibitors of VEGF action is clinically relevant especially where this concerns inhibitors whose safety has already been demonstrated in clinical trials for other indications. Our results provide a rationale for PARP inhibitors to be tested as anti-angiogenesis therapies in cancer, macular degeneration, arthritis, and other diseases characterized by excessive neovascularization.

\section{Acknowledgements}

This study was supported in part by grants from the Greek Ministry of Education and the Greek Secretariat of Research and Technology to AP, and by the US National Institutes of Health grant R01GM060915 to CS.

\section{References}

1. Conway EM, Collen D and Carmeliet P: Molecular mechanisms of blood vessel growth. Cardiovasc Res 49: 507-521, 2001.

2. Folkman J and Shing Y: Angiogenesis. J Biol Chem 267: 10931-10934, 1992.

3. Carmeliet P: Angiogenesis in health and disease. Nature Med 9: 653-660, 2003.

4. Folkman J: Angiogenesis in cancer, vascular, rheumatoid and other disease. Nat Med 1: 27-31, 1995.

5. Jagtap P and Szabó C: Poly(ADP-ribose) polymerase and the therapeutic effects of its inhibitors. Nat Rev Drug Discov 4: 421-440, 2005.

6. Szabó C, Zingarelli B, O'Connor M and Salzman A: DNA strand breakage, activation of poly(ADP-ribose) synthetase, and cellular energy depletion are involved in the cytotoxicity of macrophages and smooth muscle cells exposed to peroxynitrite. Proc Natl Acad Sci USA 93: 1753-1758, 1996.

7. Cohen-Armon M, Visochek L, Rozensal D, Kalal A, Geistrikh I, Klein R, Bendetz-Nezer S, Yao Z and Seger R: DNA-independent PARP-1 activation by phosphorylated ERK2 increases Elk1 activity: A link to histone acetylation. Mol Cell 25: 297-308, 2007.

8. Homburg S, Visochek L, Moran N, Dantzer F, Priel E, Asculai E, Schwartz D, Rotter V, Dekel N and Cohen-Armon M: A fast signal-induced activation of poly(ADP-ribose) polymerase: a novel downstream target of phospholipase c. J Cell Biol 150: 293-307, 2000.

9. Schreiber V, Dantzer F, Ame J and de Murcia G: Poly(ADPribose): novel functions for an old molecule. Nat Rev Mol Cell Biol 7: 517-528, 2006.

10. Oei S, Griesenbeck J, Schweiger M and Ziegler M: Regulation of RNA polymerase II-dependent transcription by poly(ADP-ribosyl)ation of transcription factors. J Biol Chem 273: 31644-31647, 1998.

11. Beckert S, Farrahi F, Perveen Ghani Q, Aslam R, Scheuenstuhl H, Coerper S, Konigsrainer A, Hunt TK and Hussain MZ: IGF-Iinduced VEGF expression in HUVEC involves phosphorylation and inhibition of poly(ADP-ribose) polymerase. Biochem Biophys Res Commun 341: 67-72, 2006.

12. Rajesh M, Mukhopadhyay P, Batkai S, Godlewski G, Hasko G, Liaudet L and Pacher P: Pharmacological inhibition of poly(ADP-ribose) polymerase inhibits angiogenesis. Biochem Biophys Res Commun 350: 352-357, 2006. 
13. Rajesh M, Mukhopadhyay P, Godlewski G, Batkai S, Hasko G, Liaudet L and Pacher P: Poly(ADP-ribose) polymerase inhibition decreases angiogenesis. Biochem Biophys Res Commun 350: 1056-1062, 2006

14. Jagtap P, Soriano F, Virág L, Liaudet L, Mabley J, Szabó E, Haskó G, Marton A, CB, Gallyas F Jr, Somegi B, Hoyt D, Baloglu E, VanDuzer J, Salzman A, Southan G and Szabó C: Novel phenanthridinone inhibitors of poly(adenosine 5'-diphosphate-ribose) synthetase: potent cytoprotective and antishock agents. Crit Care Med 30: 1071-1082, 2002.

15. Pyriochou A, Beis D, Koika V, Polytarchou C, Papadimitriou E, Zhou Z and Papapetropoulos A: Soluble guanylyl cyclase activation promotes angiogenesis. J Pharmacol Exp Ther 319: 663-671, 2006.

16. Miknyoczki S, Jones-Bolin S, Pritchard S, Hunter K, Zhao H, Wan W, Ator M, Bihovsky R, Hudkins R, Chatterjee S, Klein-Szanto A, Dionne $\mathrm{C}$ and Ruggeri B: Chemopotentiation of temozolomide, irinotecan, and cisplatin activity by CEP-6800, a poly(ADP-ribose) polymerase inhibitor. Mol Cancer Ther 2: 371-382, 2003.

17. Veuger S, Curtin N, Smith G and Durkacz B: Effects of novel inhibitors of poly(ADP-ribose) polymerase-1 and the DNAdependent protein kinase on enzyme activities and DNA repair. Oncogene 23: 7322-7329, 2004.

18. Plummer E: Inhibition of poly(ADP-ribose) polymerase in cancer. Curr Opin Pharmacol 6: 364-368, 2006.

19. Tentori L, Lacal PM, Muzi A, Dorio AS, Leonetti C, Scarsella M, Ruffini F, Xu W, Min W, Stoppacciaro A, Colarossi C, Wang Z-Q, Zhang J and Graziani G: Poly(ADP-ribose) polymerase (PARP) inhibition or PARP-1 gene deletion reduces angiogenesis. Eur J Cancer 43: 2124-2133, 2007.

20. McMullen M, Keller R, Sussman M and Pumiglia K: Vascular endothelial growth factor-mediated activation of p38 is dependent upon Src and RAFTK/Pyk2. Oncogene 23: 1275-1282, 2004.

21. McMullen ME, Bryant PW, Glembotski CC, Vincent PA and Pumiglia KM: Activation of p38 has opposing effects on the proliferation and migration of endothelial cells. J Biol Chem 280: 20995-21003, 2005.

22. Zachary I: VEGF signalling: integration and multi-tasking in endothelial cell biology. Biochem Soc Trans 31: 1171-1177, 2003.
23. Veres B, Gallyas F, Varbiro G, Berente Z, Osz E, Szekeres G, Szabó C and Sumegi B: Decrease of the inflammatory response and induction of the Akt/protein kinase B pathway by poly(ADP-ribose) polymerase 1 inhibitor in endotoxin-induced septic shock. Biochem Pharmacol 65: 1373-1382, 2003.

24. Veres B, Radnai B, Gallyas FJ, Varbiro G, Berente Z, Osz E and Sumegi B: Regulation of kinase cascades and transcription factors by a poly(ADP-ribose) polymerase-1 inhibitor, 4-hydroxyquinazoline, in lipopolysaccharide-induced inflammation in mice. J Pharmacol Exp Ther 310: 247-255, 2004.

25. Murohara T, Asahara T, Silver M, Bauters C, Masuda H, Kalka C, Kearney M, Chen D, Symes JF, Fishman MC, Huang PL and Isner JM: Nitric oxide synthase modulates angiogenesis in response to tissue ischemia. J Clin Invest 101: 2567-2578, 1998.

26. Ziche M, Morbidelli L, Choudhuri R, Zhang HT, Donnini S, Granger HJ and Bicknell R: Nitric oxide synthase lies downstream from vascular endothelial growth factor-induced but not basic fibroblast growth factor-induced angiogenesis. J Clin Invest 99: 2625-2634, 1997.

27. Pyriochou A, Zhou Z, Koika V, Petrou C, Cordopatis P, Sessa W and Papapetropoulos A: The phosphodiesterase 5 inhibitor sildenafil stimulates angiogenesis through a protein kinase G/MAPK pathway. J Cell Physiol 211: 197-204, 2007.

28. Fulton D, Gratton JP, McCabe TJ, Fontana J, Fujio Y, Walsh K, Franke TF, Papapetropoulos A and Sessa WC: Regulation of endothelium-derived nitric oxide production by the protein kinase Akt. Nature 399: 597-601, 1999.

29. Papapetropoulos A, Garcia-Cardena G, Madri JA and Sessa WC: Nitric oxide production contributes to the angiogenic properties of vascular endothelial growth factor in human endothelial cells. J Clin Invest 100: 3131-3139, 1997.

30. Nakajima H, Nagaso H, Kakui N, Ishikawa M, Hiranuma T and Hoshiko S: Critical role of the automodification of poly(ADPribose) polymerase-1 in nuclear factor-kappaB-dependent gene expression in primary cultured mouse glial cells. J Biol Chem 279: 42774-42786, 2004

31. Fukumura D, Gohongi T, Kadambi A, Izumi Y, Ang J, Yun C, Buerk D, Huang P and Jain R: Predominant role of endothelial nitric oxide synthase in vascular endothelial growth factorinduced angiogenesis and vascular permeability. Proc Natl Acad Sci USA 98: 2604-2609, 2001 